\title{
Salud sexual y reproductiva de hombres jóvenes en Chile: resultados de un estudio cualitativo
}

\author{
Alexandra Obach, ${ }^{1}$ Michelle Sadler, ${ }^{2}$ Francisco Aguayo ${ }^{3}$ y Margarita Bernales ${ }^{1}$
}

Forma de citar

Obach A, Sadler M, Aguayo F, Bernales M. Salud sexual y reproductiva de hombres jóvenes en Chile: resultados de un estudio cualitativo. Rev Panam Salud Publica. 2018;42:e124. https:/ / doi.org/10.26633/ RPSP.2018.124

RESUMEN Objetivo. Describir, a partir de las percepciones de adolescentes y personal de atención de salud, las formas en que los adolescentes varones se relacionan con la atención de salud sexual y reproductiva, las transformaciones percibidas en este ámbito y las estrategias desplegadas por Espacios Amigables para la atención de salud adolescente para acercarse a ellos.

Métodos. Estudio cualitativo etnográfico en Espacios Amigables para la atención de adolescentes y jóvenes de cinco municipios de la Región Metropolitana de Chile. Se utilizaron las técnicas de entrevistas semiestructuradas $(N=38)$, grupos de discusión $(N=5)$ y observación participante.

Resultados. Se identifica una falta de perspectiva de masculinidades en la atención de salud sexual y reproductiva en adolescentes, que se percibe como un ámbito enfocado principalmente para el género femenino. La mayoría de los adolescentes varones percibe a los servicios de salud como distantes y acuden principalmente en casos de emergencia. Se identifica una baja asistencia de hombres a servicios de salud sexual y reproductiva en Espacios Amigables ubicados en centros de atención primaria de salud. Sin embargo, aquellos ubicados fuera de estos como, por ejemplo, un centro exclusivo de atención de salud adolescente y servicios integrados en las escuelas, convocan a mayor número de varones.

Conclusiones. Una mayor oferta de servicios de salud sexual y reproductiva para adolescentes no necesariamente implica un aumento en el acceso de hombres. Para que esto suceda, es importante invitarlos desde temáticas que les resulten de interés, acercar los servicios de salud hacia donde ellos están, potenciar el trabajo intersectorial, e incorporar un enfoque de masculinidades para su atención.

Palabras clave Salud del adolescente; salud del hombre; atención primaria de salud; salud sexual; salud reproductiva; Chile.

Programa de Estudios Sociales en Salud, Facultad de Medicina Clínica Alemana, Universidad del Desarrollo, Santiago, Chile. Enviar correspondencia a Alexandra Obach, aobach@udd.cl

2 Departamento de Historia y Ciencias Sociales, Facultad de Artes Liberales, Universidad Adolfo Ibáñez, Santiago, Chile.

3 Escuela de Psicología, Pontificia Universidad Católica de Valparaíso, Chile.
El acceso de los varones adolescentes a servicios de salud sexual y reproductiva (SSR) se ha visto limitado por el hecho que la SSR ha sido históricamente conceptualizada como un asunto de mujeres (1-4). Por otra parte, los adolescentes varones se ven sometidos a diversos mandatos culturales que los sitúan en una posición de riesgo en SSR, entre ellas: vivir su sexualidad de acuerdo a los preceptos de la masculinidad hegemónica $(1,5)$, tener mayor número de parejas sexuales que las mujeres, tener sexo bajo la influencia del alcohol o

Este es un artículo de acceso abierto distribuido bajo los términos de la licencia Creative Commons Attribution-NonCommercial-NoDerivs 3.0 IGO, que permite su uso, distribución y reproducción en cualquier medio, siempre que el trabajo original se cite de la manera adecuada. No se permiten modificaciones a los artículos ni su uso comercial. Al reproducir un artículo no debe haber ningún indicio de que la OPS o el artículo avalan a una organización o un producto específico. El uso del logo de la OPS no está permitido. Esta leyenda debe conservarse, junto con la URL original del artículo. 
drogas y comportamientos que ponen en riesgo a otros, como resistirse al uso del preservativo $(6,7)$. Se sostiene que los hombres jóvenes hacen escaso uso de los centros de salud (8-10), y que contar con servicios amigables de salud para adolescentes, aunque es de gran importancia, no garantiza su participación (11).

Diversos estudios internacionales y en Chile describen barreras actitudinales e institucionales para el acceso de los hombres jóvenes a los servicios de SSR, entre ellas una socialización masculina de poco cuidado de su propia salud, vinculación con salud principalmente por episodios de emergencia, vergüenza de reconocer que necesitan información sobre SSR, escasa capacitación del personal de salud para la atención de hombres jóvenes y actitudes negativas hacia ellos por parte del personal de salud $(3,6,8,12,13)$.

En Chile, desde el año 2008 se vienen implementando en atención primaria los denominados Espacios Amigables para la atención de salud de adolescentes y jóvenes entre 10 y 19 años de edad (14, 15). Estos espacios tienen base en un modelo promocional y preventivo de salud, con especial énfasis en la atención de SSR, en horarios diferenciados, con respeto a la privacidad, atención amigable y personal capacitado (16). Si bien esto representa un avance, se constata que los servicios de SSR aún se centran en las mujeres: en 2014, de la población de 1519 años del país que recibió algún método anticonceptivo en la red pública de salud, $98,3 \%$ fueron mujeres y solo $1,7 \%$ hombres (17). Un estudio del año 2017 realizado en el país reporta que, de acuerdo a la percepción de los equipos de salud, existe poca visibilidad de los adolescentes y sus necesidades, y baja asistencia de ellos a los centros de salud, la cual se torna crítica en el caso de los varones (18).

El objetivo del artículo es describir la relación de los adolescentes varones con la atención de SSR, las transformaciones percibidas en este ámbito y las estrategias desplegadas por Espacios Amigables para acercarse a ellos, de acuerdo a los resultados de un estudio cualitativo etnográfico focalizado en Espacios Amigables de cinco municipios de la Región Metropolitana de Chile.

\section{MATERIALES Y MÉTODOS}

Se realizó un estudio cualitativo etnográfico, diseño escogido ya que permite comprender el sentido y significado que los actores sociales asignan a los eventos en sus contextos cotidianos $(19,20)$. El detalle de la metodología ha sido publicado (21). El trabajo de campo se llevó a cabo durante el año 2016 en cinco municipios de la Región Metropolitana de Chile: Cerro Navia, Melipilla, Pudahuel, Renca y Lo Prado, seleccionados por presentar algunas de las tasas más altas de embarazo adolescente: en el año 2015, los cinco municipios presentaron un 12,4\% de nacidos vivos de madres entre 15 y 19 años, mientras que el promedio de la Región Metropolitana fue de 8,8 (22). En estos cinco municipios operan once Espacios Amigables. Se seleccionó uno por municipio, con un total de cinco Espacios Amigables.

Las técnicas de investigación utilizadas fueron la observación participante de la dinámica de los Espacios Amigables; entrevistas semiestructuradas con informantes clave, personal de salud que trata de manera directa con adolescentes, y adolescentes usuarios y no usuarios de los Espacios Amigables; además de grupos de discusión con estos dos últimos. Todas las observaciones se registraron en diarios de campo, y las entrevistas y los grupos de discusión en audio (cuadro 1).

Los informantes clave fueron seleccionados por su experticia en la temática de estudio y por desempeñarse en áreas afines. Se incluyó una autoridad del Servicio de Salud Metropolitano Occidente (del cual dependen los municipios seleccionados), y otra a nivel comunal, un director de centro de salud, una directora de escuela y un joven líder de una organización juvenil. El diseño muestral consideró a adolescentes, de los cuales la mitad eran varones y $60 \%$ usuarios y $40 \%$ no usuarios, lo cual se logró a cabalidad. El reclutamiento del personal de salud se realizó mediante contacto directo en los Espacios Amigables, con la inclusión de todo el

\section{CUADRO 1. Entrevistas semiestructuradas y grupos de discusión}

\begin{tabular}{|c|c|c|c|}
\hline & $\begin{array}{l}\text { Entrevistas semiestructuradas } \\
\quad\left(\mathrm{N}^{\circ} \text { participantes }\right)\end{array}$ & $\begin{array}{l}\text { Grupos de discusión } \\
\left.\text { ( }{ }^{\circ} \text { participantes }\right)\end{array}$ & Total de participantes \\
\hline Informantes clave & 5 & 0 & 5 \\
\hline Personal de salud & 10 & 13 & 23 \\
\hline Adolescentes & 23 & 27 & 50 \\
\hline Total & 38 & 40 & 78 \\
\hline
\end{tabular}

a Dos grupos de discusión fueron con personal de salud y tres fueron con adolescentes.

Elaboración propia a partir de los resultados presentados. personal que trabaja con adolescentes en la atención de SSR (equipos conformados por cuatro o cinco personas). En cada Espacio Amigable se invitó a participar a dos miembros del equipo de salud en entrevistas semiestructuradas $y$, al resto, en grupos de discusión.

Los adolescentes usuarios de Espacios Amigables fueron contactados a través del personal de salud y en forma directa por el equipo de investigación en salas de espera. Se reclutó así una muestra de adolescentes tanto para las entrevistas semiestructuradas como para los grupos de discusión. Los adolescentes no usuarios fueron reclutados a través de un muestreo de bola de nieve a partir de contactos entregados por adolescentes usuarios. Se incluyó a adolescentes de entre 15 y 19 años mediante un muestreo opinático, siguiendo criterios pragmáticos y de factibilidad (20). Durante el trabajo de terreno, la muestra se intencionó con el fin de incluir a mujeres embarazadas y hombres con parejas en gestación, o que ya habían sido madres o padres, así como también adolescentes sin hijos, adolescentes de pueblos originarios y de otras nacionalidades y adolescentes que se identificaran con diferentes orientaciones sexuales.

Las notas de campo y audios fueron transcritos en forma literal. El material fue analizado mediante la estrategia de análisis temático (23), con el software $\mathrm{NVivo}^{\circledR}$.

El proyecto contó con la aprobación del Comité de Ética del Servicio de Salud Metropolitano Occidente. Todos los participantes recibieron información sobre los objetivos y procedimientos del estudio: los mayores de edad firmaron un consentimiento informado, y los menores de edad (17 años y menos) un asentimiento informado y, además, se pidió el consentimiento de sus padres. De todos los contactos, cinco adolescentes no participaron por falta de consentimiento de sus padres. 
Se realizaron un total de 38 entrevistas semiestructuradas (cinco a informantes clave, 10 a personal de salud y 23 a adolescentes) y cinco grupos de discusión (dos con personal de salud con un total de 13 participantes y tres con adolescentes con un total de 27 participantes).

Para garantizar el anonimato, se asignaron códigos que se utilizaron en las transcripciones, notas de campo y toda información analítica generada a partir del estudio.

\section{RESULTADOS}

\section{Vinculación de adolescentes varones con los servicios de salud}

La mayoría de los adolescentes varones entrevistados se acercan a los hospitales antes que a centros de atención primaria, y lo hacen por problemas graves o de riesgo vital: "Cuando yo me enfermo voy al médico, al hospital". En general, la atención de salud la significan como una instancia desagradable, que les produce miedo y evitan: "Tendría que estar muriéndome para ir al consultorio, o si no, no voy".

En la misma línea, el personal de salud relata que, de acuerdo a su experiencia, los adolescentes varones perciben al sector salud como distante. Sostienen que, durante la primera infancia, los varones acuden al sector salud para el control de niño sano, pero luego dejan de asistir. Así lo expresa una matrona entrevistada: "¿Cuándo llega el joven acá? Es cuando ya está muy enfermo digamos físicamente (...) Efectivamente, el grupo que menos se atiende con nosotros son los adolescentes varones".

A diferencia de lo anterior, adolescentes y equipos de salud coinciden en plantear que las mujeres se mantienen en contacto con el sector salud, pues en ellas se centran los cuidados y prestaciones relativas a SSR desde la adolescencia. Además, son las mujeres quienes asumen el rol de informar y vincular a los adolescentes varones con los ámbitos de salud, ya sea como madres de los varones o como parejas de estos, socializándolos en la información recibida en el área de SSR. Una adolescente lo expresa así: "Es que los hombres como que se guían por la mujer, sí, como que la mujer tiene que preguntar y después los hombres le preguntan y como que ahí se informan".

El personal de atención de salud entrevistado reconoce perpetuar una visión y práctica que centra la atención en
SSR en los cuerpos femeninos, no conceptualizar a los adolescentes varones como sujetos propios de la atención, tener actitudes negativas o prejuicios hacia ellos y contar con escaso entrenamiento para atenderlos. Como exponen dos matronas: "Yo tengo muchas colegas que dicen: 'tú atiende a los hombres porque yo no les voy a ver el pene'. Y les digo: 'pero si vemos a hombres y mujeres, hacemos salud sexual'. Y'no, no, no', dicen (...). Las colegas atendian a las puras mujeres con infecciones de trasmisión sexual ¿Y los hombres?: 'no qué asco', y no los atienden"; "A nosotras nunca nos hablaron, nunca nos enseñaron sobre cómo atender hombres, casi siempre el contacto de la matrona es con puras mujeres".

Es así como los adolescentes varones llegan excepcionalmente por su cuenta a

CUADRO 2. Características de personal de salud y adolescentes que participaron en entrevistas semiestructuradas y grupos de discusión ${ }^{a}$

\begin{tabular}{|c|c|c|}
\hline Personal de salud $(n=23)$ & $\mathrm{N}$ & $\%^{\mathrm{b}}$ \\
\hline \multicolumn{3}{|l|}{ Sexo } \\
\hline Femenino & 21 & 91,3 \\
\hline Masculino & 2 & 8,7 \\
\hline \multicolumn{3}{|l|}{ Profesión } \\
\hline Matrón/a & 9 & 39,1 \\
\hline Trabajador/a social & 6 & 26 \\
\hline Psicólogo/a & 3 & 13 \\
\hline Nutricionista & 2 & 8,7 \\
\hline Técnico/a & 2 & 8,7 \\
\hline Enfermero/a & 1 & 4,3 \\
\hline \multicolumn{3}{|l|}{ Edad (años) } \\
\hline $23-30$ & 11 & 47,8 \\
\hline $31-40$ & 8 & 34,8 \\
\hline 410 más & 4 & 17,4 \\
\hline \multicolumn{3}{|l|}{ Años trabajando con adolescentes } \\
\hline $0-1$ & 4 & 17,4 \\
\hline $1-3$ & 5 & 21,7 \\
\hline $3-6$ & 7 & 30,4 \\
\hline 60 más & 7 & 30,4 \\
\hline Adolescentes & $\mathrm{N}=50$ & $\%$ \\
\hline \multicolumn{3}{|l|}{$\overline{\text { Sexo }}$} \\
\hline Femenino & 25 & 50 \\
\hline Masculino & 25 & 50 \\
\hline \multicolumn{3}{|l|}{ Edad (años) } \\
\hline $15-16$ & 17 & 34 \\
\hline $17-19$ & 33 & 66 \\
\hline \multicolumn{3}{|l|}{ Nacionalidad/etnicidad } \\
\hline Chileno/a & 41 & 82 \\
\hline Chileno/a-mapuche & 5 & 10 \\
\hline Extranjero (peruano, ecuatoriano, colombiano) & 4 & 8 \\
\hline \multicolumn{3}{|l|}{ Orientación sexual } \\
\hline Heterosexual & 46 & 92 \\
\hline Homosexual & 3 & 6 \\
\hline Bisexual & 1 & 2 \\
\hline \multicolumn{3}{|l|}{ Uso de Espacio Amigable } \\
\hline Usuario/a & 30 & 60 \\
\hline No usuario/a & 20 & 40 \\
\hline \multicolumn{3}{|l|}{ Maternidad/paternidad } \\
\hline Sin hijos & 44 & 88 \\
\hline Un hijo & 4 & 8 \\
\hline Embarazada & 2 & 4 \\
\hline
\end{tabular}

a Se excluyen los informantes clave.

${ }^{\text {b } L o s ~ n u ́ m e r o s ~ p u e d e n ~ n o ~ s u m a r ~} 100 \%$, ya que fueron redondeados al primer decimal. Elaboración propia a partir de los resultados presentados. 
los servicios de SSR, y si lo hacen es en su mayoría para buscar preservativos.

En las entrevistas realizadas, se identificaron ciertas transformaciones respecto al modelo antes descrito, ilustradas en adolescentes con un rol más activo en el cuidado de la SSR de ellos mismos y sus parejas. El personal de salud percibe que los adolescentes varones están más informados en los ámbitos de SSR que hace algunos años: "¡Sí! Los chiquillos [adolescentes] sí se están preocupando porque cuando uno va hacer el control de joven sano al establecimiento y pregunta, los chicos sí tienen manejo de la información". A la vez, reconocen que es más frecuente verlos con sus parejas en los controles de salud, aunque muchos lo hacen como acompañantes de ellas, como plantea una matrona entrevistada: "Vienen más que antes, pero por iniciativa de las niñas, para que ellas se sientan acompañadas; no para preguntar por ellos mismos o cómo ellos se pueden cuidar". En casos menos frecuentes, como expresan adolescentes y personal de salud, los adolescentes varones son quienes promueven las visitas al consultorio con sus parejas para el uso de métodos anticonceptivos. El reporte de estos comportamientos da cuenta de una mayor conciencia y participación de los hombres en los ámbitos de SSR, aun cuando lo hagan a través de sus parejas mujeres. Tal como relata uno de los adolescentes entrevistados: "(...) es que como ella era virgen y yo no, yo le dije, 'yo no te voy a tocar mientras tú no te cuides', y me dijo 'bueno, saquemos las pastillas en el consultorio'. 'Vamos, yo te acompaño', le dije yo, y ahífuimos juntos".

Junto con lo anterior, la experiencia de paternidad es un hito que los adolescentes varones identifican como central en su acercamiento a los servicios de salud: "Nunca la acompañé a la matrona, o sea ya después cuando estaba embarazada fui con ella a todos los controles y todo, pero antes de eso, no (...). Igual es una experiencia nueva, se aprendieron cosas nuevas, procesos nuevos que yo no sabía (...) que yo nunca había ido al consultorio". El mismo joven agrega: "El ideal sería que a uno le hablaran de esas cosas y de lo que significa ser papá antes de que pase, como que uno no le toma el peso hasta que le toca".

\section{Estrategias del sector salud para involucrar a adolescentes varones en SSR}

De los cinco Espacios Amigables abordados por el estudio, tres se ubican al interior de centros de atención primaria y realizan la mayoría de sus acciones en SSR en dichos espacios, y solo algunas fuera de los establecimientos de salud, por ejemplo, charlas informativas y controles de salud en establecimientos educacionales. A través de la observación etnográfica, se pudo constatar que la mayor cantidad de usuarios de estos Espacios Amigables son mujeres, quienes principalmente buscan información y/o métodos anticonceptivos. Los hombres presentes suelen ser sus parejas, y asumen un rol de acompañantes más que de usuarios del sistema.

Las otras dos experiencias identificadas funcionan de manera diferente ya que corresponden a modelos de Espacios Amigables ubicados fuera de los centros de salud. Una de ellas es el Centro del Adolescente de Melipilla, ideada como una estrategia para la atención exclusiva de adolescentes en un espacio independiente del centro de atención primaria. En una primera instancia, este centro se enfocó en la prevención del embarazo en mujeres adolescentes. Una de las profesionales encargadas sostiene: "Se nos embarazaban mucho las chiquillas, había ingresos tardíos a control de embarazo (...) pensado en ese momento solo para las adolescentes, para las mujeres, en ningún momento nos planteamos la posibilidad de extender nuestras atenciones hacia los varones porque en realidad nuestra prioridad era la prevención del embarazo". Este modelo se fue transformando al incorporar de manera gradual a adolescentes varones. Una de las matronas del equipo comenta: "Empezó a circular otro tipo de adolescente también y eso permitió irse dando cuenta a la comunidad que no era solo era el embarazo, sino que eran todas las otras necesidades que tenían los adolescentes". Agrega: "Entonces, desde esa perspectiva nos empezó a hacer mucho ruido, el tema de cómo podíamos nosotros darle una mejor calidad de atención a los jóvenes y permitir que ellos se acercaran al centro de salud". Fue así cómo, en forma paulatina, fue apareciendo el sujeto varón adolescente en tanto usuario a través de prestaciones vinculadas a la alimentación y la salud mental. Como lo relata una psicóloga del equipo de salud: "Entonces ya no era solo la chiquilla que iba por embarazo, ya no era solo la chiquilla que iba por método anticonceptivo, sino que era la adolescente y los adolescentes, por ejemplo, que tenían problemas de mal nutrición, que tenían problemas emocionales". Esto llevó a la generación de estrategias de inserción de los hombres en las actividades de SSR del Centro, por ejemplo, en los talleres de sexualidad, los cuales en ocasiones han tenido más participantes varones que mujeres. Un matrón del Centro relata: "Nos ha pasado en un taller que terminamos solamente con hombres. Partimos el taller con veinte, eran cinco mujeres, quince hombres y terminaron viniendo solamente los hombres". Por su parte, los adolescentes dicen sentirse incorporados en este Centro y relatan estar aprendiendo que ellos también son sujetos activos en los temas relativos a SSR, como comentan dos adolescentes varones: "Como que nos abre la mente, uno tenía tantas ideas distintas, que pensaba que eran así las cosas, como que el hombre no tiene nada que meterse en estos temas, pero uno se da cuenta de que sí tiene mucho que hacer"; "Te empiezas a cuestionarte a ti mismo por qué haces las cosas, como que ahora lo piensas mejor, antes era muy arrebatado, pero ahora la piensas y piensas en las consecuencias."

La segunda estrategia corresponde al Programa 3A de la comuna de Lo Prado, el cual ha incorporado profesionales de salud al interior de las escuelas. Este programa otorga consejería y atención clínica a adolescentes en las escuelas, además de actividades en el aula. Una de las mayores fortalezas de la iniciativa de Lo Prado, de acuerdo a la percepción de los equipos de salud e informantes clave entrevistados, es que acerca a la población adolescente en general, y a los hombres en particular, a los servicios de salud ya que el personal de salud está siempre disponible para ellos. Tal como sostiene una matrona del equipo del 3A: "Como nosotras estamos permanentemente en el colegio, el hombre va a la matrona en el colegio, o sea a nosotras, que tenemos la posibilidad, la oportunidad de estar ahi, pero el hombre no va a la matrona en el consultorio". En opinión del equipo de salud, esta estrategia permite que algunos adolescentes varones perciban la atención en SSR como un derecho y responsabilidad de ellos, como lo plantea una matrona: "Yo tengo un caso de un joven y él llegaba a su casa y decía: 'mamá, yo tengo a mi matrona', y a él le hacían bullying en la casa y la mamá le decía: '¿cómo vas a tener una matrona si no estás embarazado?' (...) 'Sí, mamá, si yo tengo una matrona, una matrona', hasta que la mamá fue al colegio a conocerme, porque no entendía por qué su hijo hablaba con la matrona, así el tema cultural es tan potente". 


\section{DISCUSIÓN}

Los resultados del estudio muestran que aún existe una vinculación problemática entre los adolescentes varores y el sector salud. Esto se refleja tanto en la falta de una perspectiva específica de masculinidades en el personal de salud como en la propia percepción de los adolescentes varones, para quienes los servicios de SSR son distantes. Mientras las opiniones de algunos adolescentes varones refuerzan ideas propias de la masculinidad hegemónica, por ejemplo, que ellos solo se acercan a servicios de salud en caso de emergencia (8), se identifican ciertas transformaciones en este modelo, como una mayor asistencia de adolescentes varones a servicios de salud por temas relativos a SSR, ya sea para acompañar a sus parejas mujeres o para buscar información y atención para sí mismos. Si bien estas acciones se pueden entender como un avance en el involucramiento de los adolescentes varones en SSR, también pueden ser interpretadas como un reforzamiento de patrones tradicionales de género, ya que la SSR continúa focalizada en el cuerpo femenino. La literatura sostiene que una de las estrategias a reforzar para involucrar a los varones es aliarse con mujeres jóvenes para alentar a los hombres a acceder a servicios de SSR, sin que esto conlleve un control de ellos sobre la sexualidad de las mujeres $(6,8)$. A pesar de estas transformaciones detectadas, la percepción generalizada es que la SSR continúa centrada en las mujeres y, de esta manera, perpetúa con ello muchas de las barreras para la atención de adolescentes varones (3).

Se desprende de lo anterior que una mayor provisión de servicios de SSR para adolescentes no necesariamente implica un mayor acceso de adolescentes varones, pues dichos servicios siguen orientados sobre todo a la atención de mujeres (11). Esto se comprueba en el estudio al observar una baja asistencia de adolescentes varones a los Espacios Amigables ubicados dentro de centros de atención primaria. Por el contrario, los Espacios Amigables que se ubican fuera de los centros de salud convocan más a los varones. En la experiencia en la escuela, además de lo anterior, la disponibilidad permanente de personal de salud permite acercar los temas de SSR a los espacios cotidianos de los varones adolescentes, disminuyendo con esto sus barreras de acceso (24).

Los resultados del estudio son consistentes con lo planteado por la literatura especializada, donde se sugiere que los programas adecuados para adolescentes varones cuenten con personal de salud capaz de construir vínculos con ellos (25), se realicen en ambientes amigables y respetuosos $(6,8,15)$, ofrezcan espacios seguros para que los varones planteen sus preguntas y experiencias, los animen a tener mayor comunicación en temas de sexualidad y salud sexual con sus parejas (8), promuevan su participación a través de sus amigas y parejas mujeres (6, $8)$, los involucren desde contextos que sean relevantes para ellos (6) y formen parte de sus itinerarios cotidianos de vida, como la escuela en tanto espacio idóneo para reforzar las estrategias en SSR (26).

La principal limitación del estudio deriva de que se incluyeron solo algunos municipios de una región de Chile, por lo cual los resultados no son generalizables a la población del país. No obstante, los hallazgos son consistentes con estudios realizados tanto en Chile como en otros países de América Latina (3, 18, 24) y contribuyen a la escasa información disponible sobre el acceso de adolescentes varones a servicios de SSR.

\section{CONCLUSIONES}

En esta investigación se observaron iniciativas que, al generar espacios de atención de SSR para adolescentes fuera de los centros de atención primaria, han logrado acercar a adolescentes varones a la SSR e involucrarlos en el cuidado de su salud y la de sus parejas. De los hallazgos se desprenden algunas recomendaciones que pueden resultar de utilidad para promover y fortalecer el acceso de adolescentes varones a servicios de atención de SSR: llegar a los hombres a través de un lenguaje y temas que los convoquen como la sexualidad, la paternidad, la salud mental; acercar los servicios de salud hacia donde los hombres están, integrándose a sus contextos cotidianos; potenciar el trabajo intersectorial entre salud, educación, sociedad civil y las comunidades, e incorporar un enfoque de masculinidades para disminuir las barreras de atención en la salud de los adolescentes varones. Todas estas son acciones que permiten una vinculación efectiva y afectiva de los adolescentes varones con el sector salud, y refuerzan la idea de una atención centrada en las necesidades de los grupos específicos, en este caso, adolescentes varones en SSR.

Financiamiento Esta investigación fue posible gracias al financiamiento del Fondo Nacional en Investigación y Desarrollo en Salud FONIS (CONICYTGobierno de Chile), Proyecto FONIS \# SA15I20040.

Conflicto de intereses Ninguno declarado por los autores.

Declaración Las opiniones expresadas en este manuscrito son responsabilidad del autor y no reflejan necesariamente los criterios ni la política de la RPSP/ PAJPH y/o de la OPS.

\section{REFERENCIAS}

1. Rebello L, Gomes R. Iniciação sexual, masculinidade e saúde: narrativas de homens jovens universitários. Ciência \& Saúde Coletiva. 2009;14:653-60.

2. Pearson S. Promoting sexual health services to young men: findings from focus group discussions. Fam Plann Reprod Health Care. 2003;29(4):194-8.

3. Sadler M, Obach A, Luengo X, Biggs A. Estudio de barreras de acceso a los servicios de salud para la prevención del embarazo adolescente en Chile. Cultura Salud. Santiago de Chile; 2011.

4. Saewyc EM. What about the boys? The importance of including boys and young men in sexual and reproductive health research. J Adolesc Health. 2012;51(1):1-2.

5. Barker G, Ricardo C, Nascimento M, Olukoya A, Santos C. Questioning gender norms with men to improve health outcomes: evidence of impact. Global Public Health. 2010;5(5):539-53.
6. Garcia CM, Ptak SJ, Stelzer EB, Harwood EM, Brady SS. I connect with the ringleader: health professionals' perspectives on promoting the sexual health of adolescent males. Research in Nursing \& Health. 2014;37(6):454-65.

7. Federación Internacional de Planificación Familiar/Región del Hemisferio Occidental (IPPF/WHR por sus siglas en inglés) y Promundo. Estado de la paternidad: América Latina y el Caribe 2017. 
Nueva York: IPPF/WHR, Washington, D.C.: Promundo-US; 2017.

8. Buzi RS, Smith PB. Access to sexual and reproductive health care services: young men's perspectives. Journal of Sex \& Marital Therapy. 2014;40(2):149-57.

9. Marcell AV, Gibbs SE, Howard SR, Pilgrim NA, Jennings JM, Sanders R, et al. Do nonclinical community-based youth-serving professionals talk with young men about sexual and reproductive health and intend to refer them for care? Am J Mens Health. 2017;11(4):1046-1054.

10. Knight R, Shoveller JA, Oliffe JL, Gilbert M, Frank B, Ogilvie G. Masculinities, 'guy talk' and 'manning up': a discourse analysis of how young men talk about sexual health. Sociology of Health \& Illness. 2012;34(8):1246-61.

11. Sternberg P, Hubley J. Evaluating men's involvement as a strategy in sexual and reproductive health promotion. Health Promot Int. 2004;19(3):389-96.

12. Kalmuss D, Austrian K. Real men do... Real men don't: young Latino and African American men's discourses regarding sexual health care utilization. American Journal of Men's Health. 2010;4(3):218-30.

13. Forrest KA. Men's reproductive and sexual health. Journal of American College Health. 2001;49(6):253-66.

14. Ministerio de Salud de Chile. Servicios de salud integrales, amigables y de calidad para adolescentes, con énfasis en salud sexual y reproductiva. Orientación técnica para la atención primaria de salud. Santiago: Ministerio de Salud de Chile; 2017.

15. Hardee K, Croce-Galis M, Gay J. Are men well served by family planning programs? Reproductive Health. 2017;14(1):14.

16. Ministerio de Salud de Chile. Política Nacional de Salud de Adolescentes y Jóvenes 2008-2015. Santiago: Ministerio de Salud de Chile; 2008.

17. Dides C, Fernández C (eds.). Salud sexual y salud reproductiva y derechos humanos en Chile, Estado de la Situación 2016. Santiago: Miles Chile; 2016.

18. Rojas G, Eguiguren P, Matamala MI, Palma I, Gálvez T. Acceso a anticoncepción en adolescentes: percepciones de trabajadores de la salud en Huechuraba, Chile. Rev Panam Salud Publica. 2017;41:e77.

19. Creswell J. Research design: qualitative, quantitative, and mixed methods approaches. California: SAGE; 2014.

20. Vázquez ML, Ferreira M, Mogollón A, Frenández MJ, Delgado ME, Vargas I. Introducción a las técnicas cualitativas de investigación aplicadas en salud. Barcelona: Universidad Autónoma de Barcelona; 2005.

21. Obach A, Sadler M, Jofre N. Necesidades en salud sexual y reproductiva de adolescentes en Chile: el rol de la educación sexual. Rev Salud Publica. 2017;19(6):852-858.
22. Departamento de Estadísticas e Información en Salud (DEIS) del Ministerio de Salud de Chile. Disponible en: http://www. deis.cl/wp-content/uploads /2011/05/ SerieNacimientos_2000_2015.html

23. Thomas DR. A general inductive approach for qualitative data analysis. American Journal of Evaluation. 2006;27(2): 237-246.

24. Pinilla E, Forero C, Valdivieso MC. Servicios de salud sexual y reproductiva según los adolescentes varones (Bucaramanga, Colombia). Rev Fac Nac Salud Publica. 2009; 27(2): 164-168.

25. Koren A, Giannetti M, Hynes R, Favre $M$. Integrating male reproductive health services: one university clinic's story. Journal of Sex \& Marital Therapy. 2017; 43(1):15-23.

26. Rodríguez Vignoli J, Páez K, Ulloa C, Cox L. Reproducción en la adolescencia en Chile: la desigualdad continúa y urgen políticas activas. Santiago: CEPALUNFPA, Serie población y desarrollo $\mathrm{N}^{\circ} 116 ; 2017$.

Manuscrito recibido el 20 de noviembre de 2017. Aceptado para su publicación, tras revisión, el 29 de mayo de 2018.
ABSTRACT

\section{Sexual and reproductive health in young men in Chile: results of a qualitative study}

Keywords
Objective. To describe, based on the perceptions of adolescents and health service staff, the ways in which adolescent boys interact with sexual and reproductive health services, the changes perceived in this area over time, and the Espacios Amigables strategy ('Friendly Spaces' for adolescent health) to reach out to boys.

Methods. An ethnographic, qualitative study was conducted in Friendly Spaces for adolescent and young adult health in five municipalities belonging to Chile's Metropolitan Region. The research methods used were semi-structured interviews $(N=38)$, discussion groups $(N=5)$, and participant observation.

Results. A masculinities perspective was [TN: "un enfoque de masculinidades". Or simply: "Masculine perspectives were..."] felt to be missing in adolescent sexual and reproductive health care, which is perceived to be an area geared primarily toward females. Most adolescent boys perceive health services as distant and visit them only in case of emergency. Male attendance is low at sexual and reproductive health services in Friendly Spaces within primary health care centers. However, services outside these centers attract boys in larger numbers, for example, one that is exclusively devoted to providing adolescent health care and integrated services in schools.

Conclusions. Greater provision of adolescent sexual and reproductive health services does not necessarily translate into increased male access. For such an increase to occur, it is important to engage boys in addressing issues that capture their interest, bring health services closer to them, strengthen intersectoral work, and incorporate a masculinities perspectives in their care.

Adolescent health; men's health; primary health care; sexual health; reproductive health; Chile. 
RESUMO

\section{Saúde sexual e reprodutiva de jovens do sexo mascu- lino no Chile: resultados de um estudo qualitativo}

Objetivo. Descrever, a partir das percepções dos jovens e do pessoal de saúde, as formas como os adolescentes do sexo masculino se relacionam com a atenção de saúde sexual e reprodutiva, as transformações percebidas neste âmbito e as estratégias para atenção de saúde do adolescente por espaços amigáveis para o alcance deste grupo.

Métodos. Foi realizado um estudo qualitativo etnográfico em espaços amigáveis para atenção de saúde de adolescentes e jovens em cinco municípios da região metropolitana do Chile. Foram empregados os métodos de entrevistas semiestruturadas $(\mathrm{N}=38)$ e grupos de discussão $(\mathrm{N}=5)$ e a técnica de observação participante.

Resultados. Foi verificada a falta de uma perspectiva de masculinidades na atenção de saúde sexual e reprodutiva para adolescentes, que se percebe como sendo voltada principalmente ao sexo feminino. A maioria dos adolescentes e jovens percebe os serviços de saúde como distantes e recorrem a eles sobretudo em situações de emergência. Foi observada pouca assistência ao sexo masculino nos serviços de saúde sexual e reprodutiva em espaços amigáveis situados em unidades de atenção primária à saúde. No entanto, a frequência dos jovens é maior nos espaços localizados fora destas unidades, como em um centro exclusivo de atenção de saúde do adolescente e serviços integrados em escolas.

Conclusões. Uma maior oferta de serviços de saúde sexual e reprodutiva para adolescentes não implica necessariamente maior acesso do sexo masculino. Para isso, é importante atrair os jovens com assuntos do interesse deles, acercar os serviços de saúde de onde eles estão, reforçar o trabalho intersetorial e incorporar um enfoque de masculinidades à atenção.

Saúde do adolescente; saúde do homem; atenção primária à saúde; saúde sexual; saúde reprodutiva; Chile. 